\title{
Resistência do solo à penetração, após o tráfego com dois tipos de pneus utilizando-se um equipamento para ensaio dinâmico
}

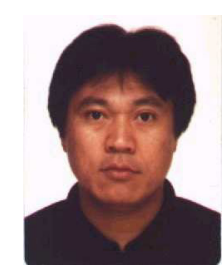

\author{
Alberto K. Nagaoka ${ }^{2}$, Kléber P. Lanças ${ }^{3}$, Pedro Castro Neto ${ }^{4}$, Afonso Lopes ${ }^{5}$ \& Saulo P. S. Guerra ${ }^{6}$ \\ 1 Parte da Tese de Doutorado do primeiro autor apresentada à FCA/UNESP, Botucatu, SP \\ 2 CAV/UDESC. CP 281, CEP 88520-000, Lages, SC. Fone: (49) 221-2211. E-mail: a2akn@cav.udesc.br (Foto) \\ 3 FCA/UNESP. CP 237, CEP 18603-970, Botucatu, SP. Telefax: (14) 6802-7118. E-mail: kplancas@fca.unesp.br \\ 4 DEG/UFLA. CP 37, CEP 37200-000, Lavras, MG. Fone: (35) 3829-1669. E-mail: pedrocn@ufla.br \\ ${ }^{5}$ FCAV/UNESP. CEP 14870-000, Jaboticabal, SP. Fone: (16) 323-2500. E-mail: afonso@fcav.unesp.br \\ 6 FCA/UNESP. CP 237, CEP 18603-970, Botucatu, SP. Telefax: (14) 6802-7118. E-mail: ssguerra@terra.com.br
}

Protocolo 82 - 14/6/2002 - Aprovado em 10/6/2003

\begin{abstract}
Resumo: Com este trabalho objetivou-se, principalmente, avaliar a resistência do solo à penetração, em função do tipo de pneu (diagonal e radial) e da carga vertical, utilizando-se um equipamento de ensaio de pneu agrícola individual, e verificar os efeitos nas camadas do solo pela passagem do pneu. $O$ equipamento utilizado foi projetado para ser acoplado na traseira do trator e acionado pela tomada de potência afim de realizar ensaios de pneus em condições de campo, sendo ainda, constituído, de uma caixa de transmissão mecânica de trator e instrumentos eletrônicos de medição. Este trabalho foi desenvolvido no Núcleo de Engenharia Rural da Faculdade de Ciências Agronômicas (FCA/UNESP) em Botucatu. Os dados obtidos no experimento foram analisados considerando-se o delineamento experimental em blocos, no esquema de parcelas subdivididas, tendo na parcela os pneus (radial e diagonal) e na subparcelas as cargas (10.000, $13.000,16.000$ e $19.000 \mathrm{~N}$ ) com cinco repetições. $\mathrm{O}$ pneu radial mostrou vantagem em relação ao diagonal, por apresentar menores valores de índice de cone. As cargas mais elevadas provocaram maiores valores de índice de cone e seus incrementos, nas camadas de 0 a 10 e de 10 a $20 \mathrm{~cm}$.
\end{abstract}

Palavras-chave: índice de cone, compactação do solo, pneu agrícola

\section{Soil penetration resistance after the traffic with two types of tyres using the dynamic test equipment}

\begin{abstract}
The main objective of this research was to evaluate the soil cone index results as a function of tyre type (radial and bias tyres) and tyre load using a single wheel testing equipment and verifying the effect on the layers after tyre traffic. This equipment was projected for linking at the rear of a tractor and was activated by the power with the purpose of testing individual tyre under field conditions and constituted of a mechanical transmission box and electronic instruments of measurement. This study was conducted at the Agricultural Tyre Testing Center - NEMPA of Rural Engineering Department, Faculty of Agronomic Sciences, UNESP, Botucatu, SP. The data obtained were analyzed considering a random block experimental design in split plot with tyre in the main plot (radial and bias tyres) and tyre load $(10.000 \mathrm{~N}, 13.000 \mathrm{~N}, 16.000 \mathrm{~N}$ and 19.000 $\mathrm{N})$ in the split plots with five repetitions. The radial tyre showed advantage in relation to bias tyre showing small cone index values. The highest loads provoked largest cone index values and its increments in the layers of 0 to $10 \mathrm{~cm}$ and 10 to $20 \mathrm{~cm}$.
\end{abstract}

Key words: cone index, soil compaction, agricultural tyre

\section{INTRODUÇÃO}

A partir da Segunda Grande Guerra Mundial, o avanço da engenharia contribuiu para o incremento na potência dos tratores e, com isto, os estudos se intensificaram na busca de máquinas e sistemas mais eficientes de tração, como é o caso dos tratores com tração nas quatro rodas. Trabalhos realizados mostraram que o peso e a potência dos tratores têm aumentado ao longo do tempo, embora a relação entre os dois fatores tenha diminuído, resultando em prejuízos ao solo e ao desempenho do trator (Cordeiro, 1994).

A movimentação de veículos e máquinas agrícolas, geralmente bastante pesados, e o preparo intensivo do solo, têm contribuído para a formação de camadas compactadas (Ferreira, 
1993; Camargo \& Alleoni, 1997; Torres et al. 1998; Castro Neto et al. 1999). Essas camadas, além de provocarem erosão superficial do solo, refletem na produtividade agrícola devendo, portanto, haver uma importante relação entre solo/máquina/ planta. Ao pesquisador cabe a função de investigar os efeitos causados pelo uso da máquina, comparar e recomendar o uso correto para que os equipamentos tenham o máximo rendimento com mínimo prejuízo.

Ao realizarem um levantamento de solos no Estado do Rio Grande do Sul, Souza et al. (1988) encontraram as camadas adensadas em profundidades que variaram entre 10 e $30 \mathrm{~cm}$, sendo as principais diferenças destas em relação às outras camadas do perfil do solo, as maiores densidades e resistência à penetração. Os mesmos autores enfatizaram que as conseqüências mais imediatas do aparecimento dessas camadas são a redução da capacidade de infiltração da água das chuvas, maior resistência à penetração das raízes das plantas, excesso de umidade acima da camada nos períodos chuvosos e deficiência de água nos períodos secos.

Marshall \& Raney (1960) definem as zonas compactadas do solo como sendo o resultado da deterioração da sua estrutura, tendo como principal conseqüência a diminuição da porosidade nas camadas logo abaixo da profundidade de trabalho dos órgãos ativos das máquinas. Os autores classificaram os solos com pouca matéria orgânica ou estrutura "fraca", como mais susceptíveis à compactação pelo tráfego e ação das máquinas e implementos.

Apesar das limitações, segundo Lanças (1991), a resistência à penetração é freqüentemente usada para a indicação comparativa do nível da compactação, em solos de mesmo tipo e mesmo teor de água, devido à facilidade e rapidez com a qual numerosas medidas podem ser realizadas. Os resultados são normalmente expressos em termos de força por unidade de área do cone de dimensões padronizadas, instalado na ponta da haste do penetrômetro ou penetrógrafo.

A avaliação da resistência mecânica do solo à penetração de uma haste é uma maneira rápida e fácil de medir a resistência à penetração a várias profundidades, sendo esta metodologia muito utilizada para se avaliar, entre outros fatores: a resistência ao rolamento (Brixius, 1987) e a compactação em diferentes sistemas de preparo do solo (Ortolani et al., 1992), predizer a força de tração de máquinas (Wolf et al., 1996) e relacionar fatores de resistência do solo à elongação radicular (Camargo \& Alleoni, 1997).

Lanças \& Santos (1998) construíram um penetrômetro hidráulico-eletrônico para a determinação da resistência à penetração do solo. O sistema tem como elementos básicos um pistão hidráulico, uma célula de carga, uma haste de aço contendo uma ponteira cônica de ângulo sólido de $30^{\circ}$, com diâmetro de 19,05 mm. O sistema hidráulico do trator ("controle remoto") foi utilizado para obtenção da pressão necessária para cravar o cone na direção normal (perpendicular) ao solo. A velocidade constante de $1820 \mathrm{~mm} \mathrm{~min}^{-1}$, independentemente da resistência do solo, foi obtida pela instalação de uma válvula de controle de vazão no circuito hidráulico. A obtenção, o registro e o armazenamento dos dados de resistência à penetração do solo foram possíveis pela utilização de sistema eletrônico formado pelos seguintes componentes: sensor de força (célula de carga), potenciômetro utilizado como sensor de profundidade, sistema de aquisição e armazenamento de dados ("Micrologger"), microcomputador e programa para análise dos dados ou planilha eletrônica. Recentemente, este penetrômetro foi adaptado para ser acoplado na traseira do chassi do trator, por Castro Neto (2001).

Fernandes (1996) realizou uma pesquisa com máquinas de exploração florestal com a finalidade de avaliar a compactação do solo causada pelo tráfego e determinar os dias potencialmente úteis para o trabalho. Utilizando-se um penetrógrafo manual para obter os dados de compactação do solo; o autor avaliou as diferenças entre a resistência à penetração pela fórmula:

$$
\mathrm{Y}=\mathrm{D}-\mathrm{A}
$$

em que:

Y - diferença entre as resistências $(\mathrm{MPa})$

$\mathrm{D}$ - resistência à penetração depois do tráfego $(\mathrm{MPa})$

A - resistência à penetração antes do tráfego $(\mathrm{MPa})$

Este autor verificou, ainda, que o maior incremento da resistência à penetração, devido ao tráfego das máquinas pesquisadas, ocorreu para a profundidade de 0 a $15 \mathrm{~cm}$.

Seraut (1990) estudou o pneu 20.8R38, suportando $39 \mathrm{kN}$, inflado com pressão de 80 e 160 kPa e constatou que a pressão na superfície do solo é semelhante à pressão de inflação do pneu e, ainda, que a pressão dentro do solo, a $30 \mathrm{~cm}$, tem relação direta com aquela usada no pneu.

Devido à disposição das lonas nos pneus radiais, ocorre redução da deflexão das garras e, por outro lado, aumenta-se a deformação lateral do pneu, resultando numa área maior de contato com o solo. Utilizando-se pressão e carga correta neste tipo de rodado, aumenta-se a deformação lateral sem comprometer sua vida útil, reduzem-se os efeitos de vibrações ou saltos ("power hop") para tratores com tração nas quatro rodas (Deere, 1993 e Goodyear Tire \& Rubber, 1994).

Ngunjiri \& Siemens, citados por Al-Adawi \& Reeder (1996), estudaram o efeito do tráfego de um trator com 8,5 t em quatro tratamentos de compactação: sem tráfego, com tráfego nas entrelinhas, com tráfego nas linhas e com tráfego em toda a área. Os autores concluíram que a produção de milho na área com tráfego em toda a área foi significativamente menor que a produção nos demais tratamentos.

Ferreira (1998) também pesquisou a compactação do solo por tráfego de máquinas de colheita no plantio florestal e concluiu que a resistência à penetração do solo foi maior para profundidade entre 5 a $15 \mathrm{~cm}$ e, também, ocorreu um incremento de aproximadamente 55\% na resistência à penetração do solo, entre a fase de pré-colheita e pós-colheita, variando de 1075,2 $\mathrm{kPa}$ para $1666,4 \mathrm{kPa}$, respectivamente.

O objetivo deste trabalho foi avaliar a resistência do solo à penetração em função do tipo de pneu (diagonal e radial) e da carga vertical, verificando-se o efeito nas camadas do solo pela passagem do pneu utilizando-se um equipamento de ensaio de pneu agrícola individual. 


\section{MATERIAL E MÉTODOS}

Os ensaios de campo foram realizados na Pista de Ensaio do NEMPA, localizado na Fazenda Experimental Lageado, da Faculdade de Ciências Agronômicas (FCA) da Universidade Estadual Paulista (UNESP) nas coordenadas geográficas $22^{\circ}$ 49' latitude Sul e $48^{\circ} 25^{\prime}$ longitude Oeste de Greenwich, com altitude média de $770 \mathrm{~m}$. O solo da área experimental foi classificado como Latossolo Roxo (Cordeiro, 2000).

A avaliação da resistência do solo à penetração antes do tráfego foi realizada em três áreas representativas do experimento, obtendo-se cinco parcelas para cada área (a média de 2 pontos amostrais constituiu uma parcela). Os valores de resistência do solo à penetração foram analisados em quatro camadas do solo ( 0 a 10, 10 a 20, 20 a 30 e 30 a $40 \mathrm{~cm}$ ) separadamente, considerando-se o delineamento experimental em blocos. Para avaliar a resistência do solo à penetração após o tráfego como um indicativo da compactação do solo, foram determinados os valores de seis pontos amostrais para cada passagem do pneu (cuja média dos seis pontos constituiu uma parcela) sob 5 níveis de cargas em 4 camadas do solo. Os índices de cone, obtidos no experimento, foram analisados considerando-se o delineamento experimental em blocos, no esquema de parcelas sub-subdivididas, tendo-se na parcela os pneus (radial e diagonal), na subparcela as cargas $(0,10.000$, $13.000,16.000$ e $19.000 \mathrm{~N}$ ) e na sub-subparcela as camadas ( 0 a 10,10 a 20, 20 a 30 e 30 a 40 cm) com cinco repetições (Figura 1).

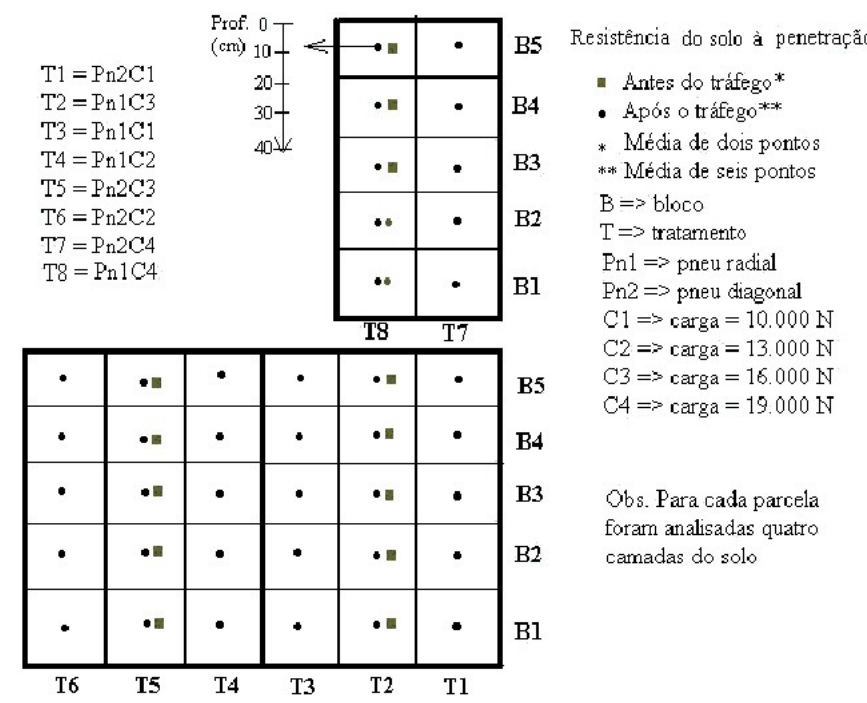

Figura 1. Diagrama da área experimental com as parcelas da resistência do solo à penetração, antes do tráfego e após o trafego dos pneus

No momento em que foi realizada a obtenção da resistência do solo à penetração, foram coletadas amostras de solo em oito pontos da área experimental, estratificada de 0 a 15 e de 15 a $30 \mathrm{~cm}$ de profundidade. As amostras foram coletadas com auxílio de um enxadão e uma pá de jardinagem, e colocadas em latinhas de alumínio, com identificação, as quais foram transportadas imediatamente para o Laboratório de Física do Solo do NEMPA, onde foi realizada a análise do teor de água do solo, seguindo-se a metodologia da EMBRAPA (1979).
Avaliaram-se a resistência do solo à penetração, em função das variáveis estudadas, e os incrementos do índice de cone, seguindo-se a metodologia de Fernandes (1996) e Castro Neto et al. (1999).

Os resultados do experimento foram interpretados estatisticamente por meio da análise de variância, adotando-se o nível de significância de $5 \%$, e pelo teste de comparação de médias de Tukey, conforme recomendação de Gomes (1987) e Curi (1997).

Para o levantamento dos dados utilizou-se um equipamento para ensaio dinâmico de rodado agrícola individual, um trator SLC-John Deere ${ }^{1}$ modelo 6600, com tração dianteira auxiliar, potência de $89 \mathrm{~kW}$ no motor e massa de $5110 \mathrm{~kg}$ sem lastro, acoplado ao equipamento, devidamente regulado e instrumentado, além de um sistema de aquisição de dados "Micrologger" CR23X (Figura 2), balança eletrônica, penetrômetro hidráulicoeletrônico (Figura 3) e dois tipos de pneu de tração (Figura 4) do tipo radial (620/75 R30(23.1-R30) e diagonal (23.1-30) (Tabela 1).

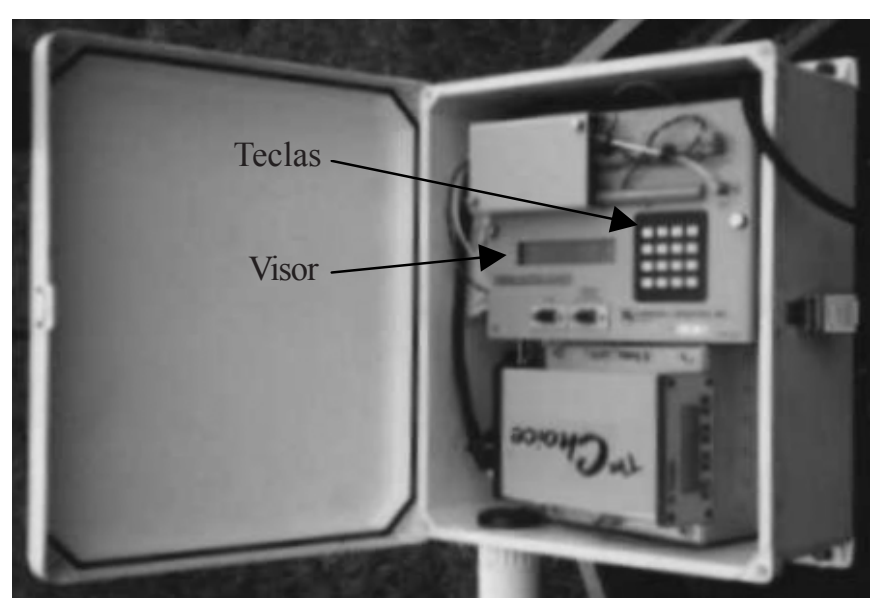

Figura 2. Sistema de aquisição de dados ("Micrologger”) utilizado no equipamento

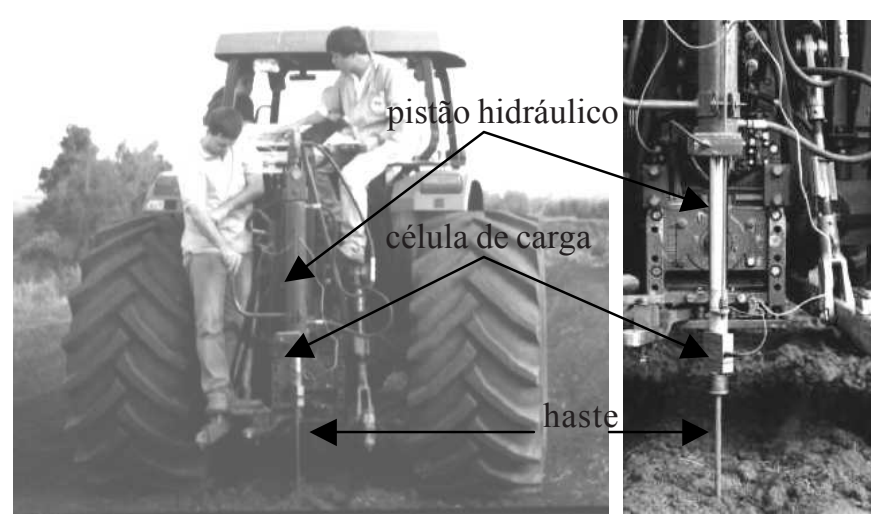

Figura 3. Penetrômetro hidráulico-eletrônico utilizado no experimento para avaliar a compactação do solo

Utilizou-se um penetrômetro hidráulico eletrônico montado no trator, desenvolvido por Castro Neto (2001), e um sistema de processamento de dados elaborado por Castro Neto et al. (1999) para importar os dados coletados no sistema de

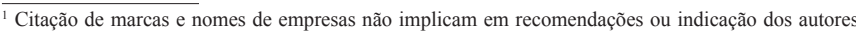


A.

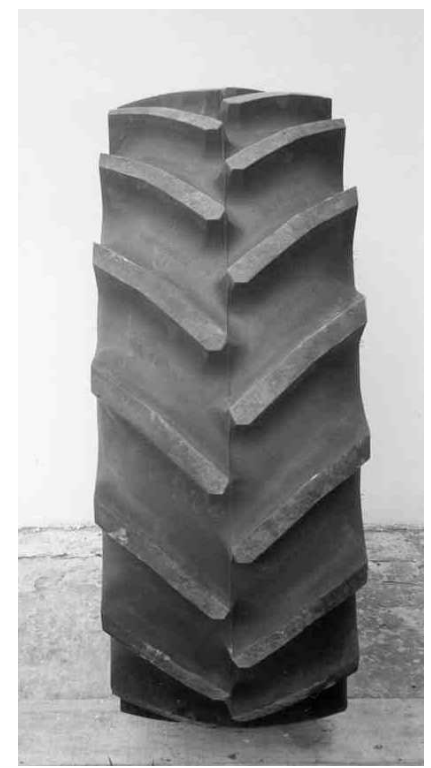

Figura 4. Vista frontal do pneu radial (A) e do pneu diagonal (B) utilizados no experimento

Tabela 1. Características técnicas dos pneus utilizados no experimento com o equipamento

\begin{tabular}{lcc}
\hline Características & Pneu Radial & Pneu Diagonal \\
\hline Dimensão & $620 / 75$ & $23.1-30$ \\
Lona/LI* & R 30 (23.1-R30) & \\
Modelo & 8 & 12 \\
Profundidade da garra (mm) & DT 820 & Dyna Torque II R1 \\
Aro & 55 & 40 \\
Diâmetro (mm) & DW20A & DW20A \\
Largura da seção (mm) & 1710 & 1707 \\
Circunferência de & 595 & 587 \\
rodagem (mm) & 5090 & 5029 \\
Raio de carga estática (mm) & 771 & 775 \\
Pressão máxima de montagem & $242 \mathrm{kPa}(35$ PSI) & $242 \mathrm{kPa}(35$ PSI) \\
Pressão de uso & $110 \mathrm{kPa}(16$ PSI) & $124 \mathrm{kPa}(18$ PSI) \\
\hline
\end{tabular}

* Índice de carga

aquisição de dados do penetrômetro, efetuar a consistência e obter valores de índice de cone do solo.

O equipamento utilizado permite que se avalie o desempenho dinâmico de rodados pneumáticos de tração, em condições de solo agrícola e em pista asfáltica; permite, também, compararse diferentes pneus em função da pressão de inflação, velocidade, patinagem, raio de rolamento e carga no rodado, e verificar o efeito da passagem do pneu no solo, conforme as condições desejadas na pesquisa. A Figura 5 apresenta o equipamento utilizado neste experimento.

\section{RESULTADOS E DISCUSSÃO}

Os ensaios foram realizados na pista de ensaio sobre solo solto, utilizando-se pneus radial e diagonal em condições diferentes de lastragem. O teor de água do solo no momento da avaliação era de $15,57 \%$ na profundidade de 0 a $15 \mathrm{~cm}$ e de $17,60 \%$ na profundidade de 15 a $30 \mathrm{~cm}$.

A análise do índice de cone do solo antes do tráfego (carga $=0 \mathrm{~N}$ ), em três áreas amostradas está apresentada na Tabela 2 ,

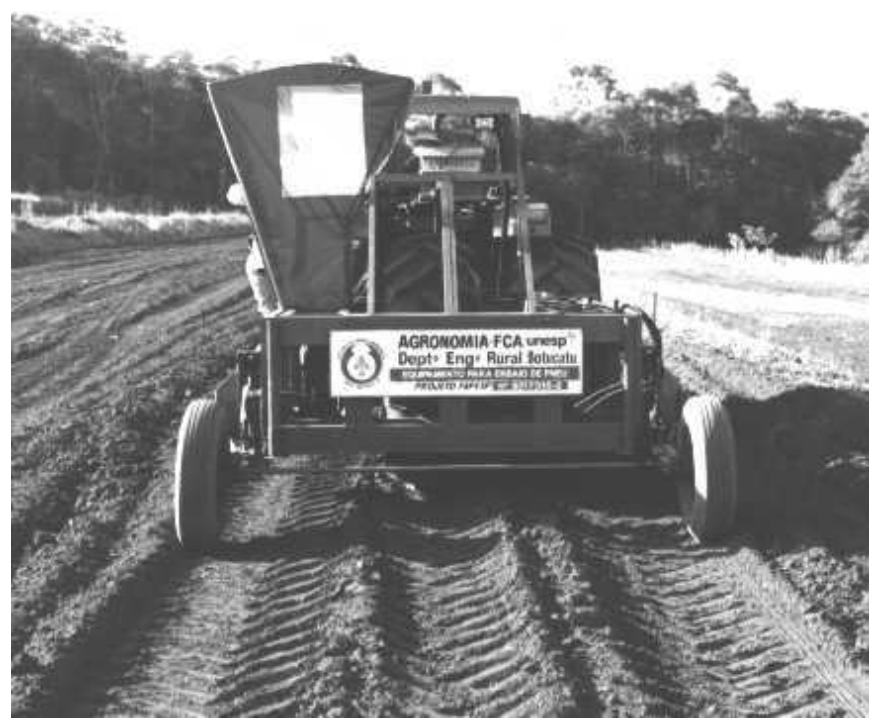

Figura 5. Equipamento para ensaio dinâmico de rodado agrícola individual utilizado no experimento

na qual se verifica que não houve diferenças significativas entre as áreas, nas camadas do solo de 0 a 10,10 a 20,20 a 30 e 30 a $40 \mathrm{~cm}$; portanto, para se comparar a resistência do solo à penetração antes e depois do tráfego, considerou-se a média dos valores das três áreas como o índice de cone para carga $0 \mathrm{~N}$.

Tabela 2. Análise de variância (quadrado médio) referente ao índice de cone antes do tráfego dos pneus $($ carga $=0 \mathrm{~N})$, em três áreas, nas profundidades de 0 a 10, 10 a 20, 20 a 30 e 30 a $40 \mathrm{~cm}$

\begin{tabular}{|c|c|c|c|c|c|}
\hline \multirow{3}{*}{$\begin{array}{c}\text { Causas da } \\
\text { Variação }\end{array}$} & \multirow{3}{*}{ GL } & \multicolumn{4}{|c|}{ Quadrado Médio } \\
\hline & & \multicolumn{4}{|c|}{ Profundidade $(\mathrm{cm})$} \\
\hline & & 0 a 10 & 10 a 20 & 20 a 30 & 30 a 40 \\
\hline Áreas & 2 & $1198,12^{\mathrm{ns}}$ & $219930,79^{\mathrm{ns}}$ & $642143,39^{\mathrm{ns}}$ & $1034824,92^{\mathrm{ns}}$ \\
\hline Bloco & 4 & $3578,50^{\mathrm{ns}}$ & $764447,96^{\mathrm{ns}}$ & $825835,67^{\mathrm{ns}}$ & $195522,18^{\mathrm{ns}}$ \\
\hline Resíduo & 8 & 6420,96 & 373383,65 & 676196,78 & 467672,90 \\
\hline $\mathrm{CV}$ & & 41,90 & 72,38 & 28,51 & 20,23 \\
\hline $\begin{array}{l}{ }^{\text {ns }} \text { Não significat } \\
* \text { * Significativo } \\
\text { ** Significativo } \\
\text { CV - Coeficient } \\
\text { GL - Graus de }\end{array}$ & $\begin{array}{l}\text { vo }(p \\
p<0,0 \\
(p<0, \\
\text { e de vi }\end{array}$ & $\begin{array}{l}0,05) \\
5) \\
\text { O1) } \\
\text { riação (\%) } \\
\text { le }\end{array}$ & & & \\
\hline
\end{tabular}

Verifica-se, na Tabela 3, que o tipo e a carga no pneu, a profundidade da camada e a interação desses fatores influenciaram nos valores de índice de cone, indicando variação na resistência do solo à penetração, devido ao tráfego dos pneus e à profundidade do solo.

Observa-se, na Tabela 4, que o índice de cone, depois do tráfego com pneu diagonal, foi maior que com pneu radial. Essas diferenças confirmam os resultados encontrados pela John Deere (1993), Goodyear Tire \& Rubber (1994) e Seraut (1990). Ressalta-se que, de acordo com as recomendações do fabrican-te, a pressão de inflação do pneu diagonal (124 kPa) foi maior que a pressão do pneu radial $(110 \mathrm{kPa})$.

Todas as cargas modificaram a condição inicial do solo, sendo que a carga de $13.000 \mathrm{~N}$ causou menor compactação do solo, como verificado por Marshall \& Raney (1960); já as camadas de 20 a 30 e de 30 a $40 \mathrm{~cm}$ apresentaram maiores valores de índice de 
cone, seguidas das camadas de 10 a 20 e de 0 a $10 \mathrm{~cm}$, confirmando os resultados obtidos por Souza et al. (1988).

Tabela 3. Resultado da análise de variância (quadrado médio) referente ao índice de cone com dois tipos de pneu (radial e diagonal), submetidos a cinco cargas: $0,10.000,13.000,16.000$ e $19.000 \mathrm{~N}$, nas profundidades de 0 a 10,10 a 20, 20 a 30 e 30 a $40 \mathrm{~cm}$

\begin{tabular}{|c|c|c|}
\hline Causas da Variação & GL & Quadrado Médio \\
\hline Pneu & 1 & $847986,13^{*}$ \\
\hline Carga & 4 & $5947913,73^{* *}$ \\
\hline Pneu x Carga & 4 & $771839,31^{\mathrm{ns}}$ \\
\hline Camada & 3 & $104632994,85^{* *}$ \\
\hline Pneu x Camada & 3 & $759244,43^{*}$ \\
\hline Carga x Camada & 12 & $971993,18^{* *}$ \\
\hline Pneu x Carga x Camada & 12 & $420978,53^{*}$ \\
\hline Bloco & 4 & $209808,93^{\mathrm{ns}}$ \\
\hline Resíduo (a) & 4 & 40445,50 \\
\hline Resíduo (b) & 32 & 392192,05 \\
\hline Resíduo (c) & 120 & 221222,04 \\
\hline $\mathrm{CV}(\mathrm{a})$ & & 8,23 \\
\hline $\mathrm{CV}(\mathrm{b})$ & & 25,61 \\
\hline $\mathrm{CV}(\mathrm{c})$ & & 19,24 \\
\hline
\end{tabular}

Tabela 4. Médias* dos valores de índice de cone nas profundidades de 0 a 10, 10 a 20, 20 a 30 e 30 a $40 \mathrm{~cm}$, de dois tipos de pneu submetidos a cinco níveis de carga(C): 0, 10.000, 13.000, 16.000 e $19.000 \mathrm{~N}$

\begin{tabular}{lc}
\hline Causas da Variação & Média do Índice de Cone $(\mathrm{kPa})$ \\
\hline Pneu Diagonal & $2510,16 \mathrm{a}$ \\
Pneu Radial & $2379,93 \mathrm{~b}$ \\
Carga $19.000 \mathrm{~N}$ & $2683,49 \mathrm{ab}$ \\
Carga $16.000 \mathrm{~N}$ & $2808,43 \mathrm{a}$ \\
Carga $13.000 \mathrm{~N}$ & $2350,16 \mathrm{~b}$ \\
Carga $10.000 \mathrm{~N}$ & $2557,91 \mathrm{ab}$ \\
Carga 0 N & $1825,25 \mathrm{c}$ \\
Camada $30-40 \mathrm{~cm}$ & $3645,20 \mathrm{a}$ \\
Camada $20-30 \mathrm{~cm}$ & $3503,02 \mathrm{a}$ \\
Camada $10-20 \mathrm{~cm}$ & $2080,41 \mathrm{~b}$ \\
Camada $0-10 \mathrm{~cm}$ & $551,55 \mathrm{c}$ \\
\hline
\end{tabular}

*Médias seguidas de mesma letra na coluna não diferem pelo teste de Tukey, em $5 \%$ de probabilidade

As Figuras 6 e 7 mostram, com maiores detalhes, o comportamento do índice de cone com o tipo de pneu e com as cargas aplicadas no pneu de 0 a $40 \mathrm{~cm}$ de profundidade.

A Tabela 5 mostra diferenças significativas nos valores de índice de cone entre as camadas do solo com tráfego de pneu radial e, também, com o pneu diagonal. Observa-se que o tipo de pneu utilizado influenciou nos valores de índice de cone para a camada de 20 a $30 \mathrm{~cm}$.

Nota-se, na Tabela 6, que a camada de 20 a $40 \mathrm{~cm}$ sofreu maior compactação do solo para ambos os pneus; no entanto, na camada de 20 a $30 \mathrm{~cm}$, o pneu diagonal ocasionou maior compactação. Seraut (1990) afirmou que a pressão na superfície do solo é semelhante à pressão de inflação do pneu e que a pressão dentro do solo, a $30 \mathrm{~cm}$, tem relação direta com aquela usada no pneu, cujos resultados encontrados confirmaram a observação feita pelo autor.
Resistência à Penetração $(\mathrm{kPa})$

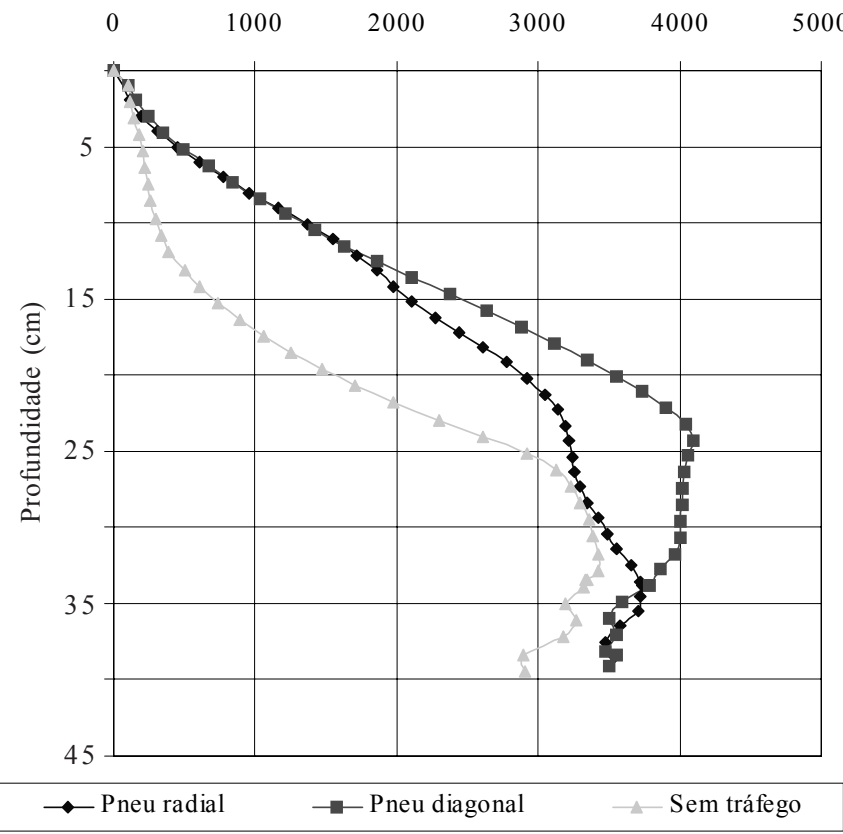

Figura 6. Resistência do solo à penetração quanto ao tráfego com pneu radial, diagonal e sem tráfego, na profundidade de 0 a $40 \mathrm{~cm}$

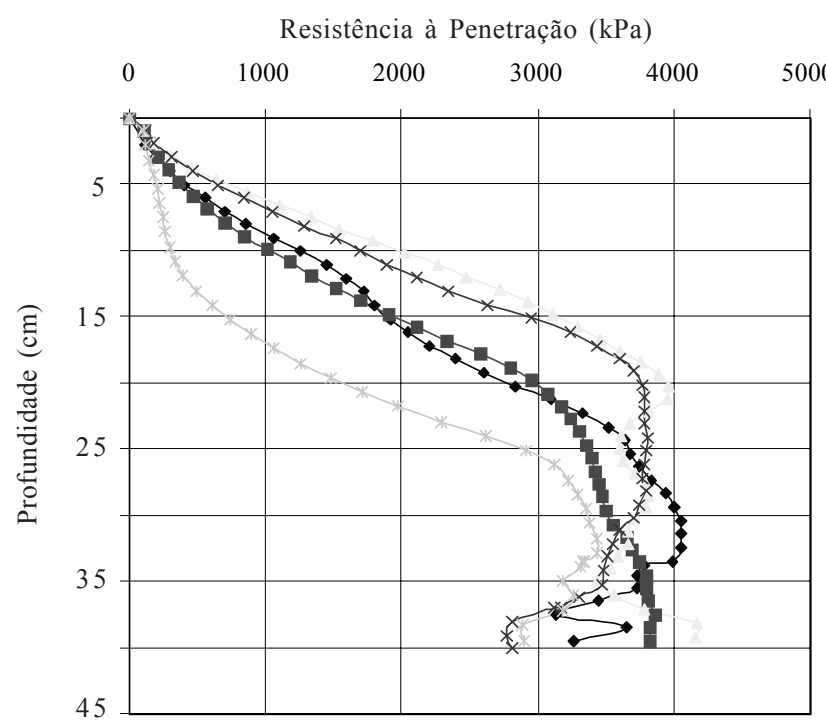

$\multimap$ Carga $10.000 \mathrm{~N} \rightarrow$ Carga $13.000 \mathrm{~N}$ $*$ Carga $19.000 \mathrm{~N} \quad$ * Sem tráfego

Figura 7. Resistência do solo à penetração quanto à carga nos pneus, na profundidade de 0 a $40 \mathrm{~cm}$

Os valores de índice de cone foram maiores na camada de 10 a $20 \mathrm{~cm}$ que na camada de 0 a $10 \mathrm{~cm}$, para ambos os pneus.

A Tabela 7 mostra que, em todas as camadas do solo estudadas, os valores de índice de cone foram influenciados pelas cargas utilizadas no pneu, além de diferenças significativas nos valores de índice de cone entre as cargas, nas camadas de 10 a 20 e 20 a $30 \mathrm{~cm}$.

Observa-se na Tabela 8 que, nas camadas de 0 a 10 e de 30 a $40 \mathrm{~cm}$, a carga utilizada não influenciou nos valores de índice de cone, mas nas camadas de 10 a $20 \mathrm{~cm}$ a compactação do solo foi maior para a carga de 16.000 e 19.000 N. Para a camada 
Tabela 5. Desdobramento da análise de variância (quadrado médio) referente ao índice de cone em cinco camadas (0 a 10, 10 a 20, 20 a 30 e 30 a $40 \mathrm{~cm}$ ) com dois tipos de pneu

\begin{tabular}{lcc}
\hline Causa da Variação & GL & Quadrado Médio \\
\hline Camadas x pneu radial & 3 & $45989767,49^{* *}$ \\
Camadas x pneu diagonal & 3 & $59402471,79^{* *}$ \\
Pneus x camada 0 a $10 \mathrm{~cm}$ & 1 & $147618,96^{\mathrm{ns}}$ \\
Pneus x camada 10 a $20 \mathrm{~cm}$ & 1 & $40533,88^{\mathrm{ns}}$ \\
Pneus x camada 20 a $30 \mathrm{~cm}$ & 1 & $2817885,77^{* *}$ \\
Pneus x camada 30 a $40 \mathrm{~cm}$ & 1 & $119680,82^{\mathrm{ns}}$ \\
\hline
\end{tabular}

ns Não significativo $(\mathrm{p}>0,05)$

* Significativo $(\mathrm{p}<0,05)$

** Significativo $(\mathrm{p}<0,01)$

GL - Graus de liberdade

Tabela 6. Médias* dos valores de índice de cone $(\mathrm{kPa})$ resultantes da interação entre camadas do solo (0 a 10, 10 a 20, 20 a 30 e 30 a $40 \mathrm{~cm}$ ) e tipo de pneu (radial e diagonal)

\begin{tabular}{lrr}
\hline Causas da Variação & \multicolumn{2}{c}{ Pneu } \\
\cline { 2 - 3 } Camada $(\mathrm{cm})$ & Radial & Diagonal \\
\hline $30-40$ & $3596,28 \mathrm{aA}$ & $3694,13 \mathrm{aA}$ \\
$20-30$ & $3265,63 \mathrm{aB}$ & $3740,42 \mathrm{aA}$ \\
$10-20$ & $2051,94 \mathrm{bA}$ & $2108,89 \mathrm{bA}$ \\
$0-10$ & $605,88 \mathrm{cA}$ & $497,21 \mathrm{cA}$ \\
\hline
\end{tabular}

"Em cada coluna e para cada fator, médias seguidas de mesma letra minúscula não diferem pelo teste de Tukey, a $5 \%$ de probabilidade, em cada linha e para cada fator, médias seguidas de mesma letra maiúscula

Tabela 7. Desdobramento da análise de variância (quadrado médio) referente ao índice de cone em quatro camadas ( 0 a 10 , 10 a 20,20 a 30 e 30 a $40 \mathrm{~cm})$ para cinco cargas no pneu $(0$, $10.000,13.000,16.000$ e $19.000 \mathrm{~N})$

\begin{tabular}{|c|c|c|}
\hline Causa da Variação & GL & Quadrado Médio \\
\hline Camadas $\mathrm{x}$ carga $0 \mathrm{~N}$ & 3 & $23917777,51^{* *}$ \\
\hline Camadas x carga $10.000 \mathrm{~N}$ & 3 & $23328547,97^{* *}$ \\
\hline Camadas x carga $13.000 \mathrm{~N}$ & 3 & $21056549,29^{* *}$ \\
\hline Camadas x carga $16.000 \mathrm{~N}$ & 3 & $20808747,75^{* *}$ \\
\hline Camadas x carga $19.000 \mathrm{~N}$ & 3 & $19409345,05^{* *}$ \\
\hline Cargas x camada 0 a $10 \mathrm{~cm}$ & 4 & $546717,22^{\text {ns }}$ \\
\hline Cargas x camada 10 a $20 \mathrm{~cm}$ & 4 & $6189330,99^{* *}$ \\
\hline Cargas x camada 20 a $30 \mathrm{~cm}$ & 4 & $1646565,94^{* *}$ \\
\hline Cargas x camada 30 a $40 \mathrm{~cm}$ & 4 & $481279,12^{\mathrm{ns}}$ \\
\hline
\end{tabular}

de 20 a $30 \mathrm{~cm}$, todas as cargas compactaram o solo após o seu tráfego, com exceção da carga de $13.000 \mathrm{~N}$.

Nas camadas de 0 a 10, 10 a 20 e de 20 a 40 cm, os valores do índice de cone foram maiores, respectivamente, para todas as cargas no pneu.

Tabela 8. Médias* dos valores de índice de cone resultantes da interação entre camadas do solo ( 0 a 10, 10 a 20, 20 a 30 e 30 a $40 \mathrm{~cm})$ e carga aplicada no pneu $(0,10.000,13.000,16.000 \mathrm{e}$ $19.000 \mathrm{~N})$

\begin{tabular}{cccccc}
\hline \multirow{2}{*}{$\mathrm{CV}$} & \multicolumn{5}{c}{ Carga $(\mathrm{N})$} \\
\cline { 2 - 6 } & 19.000 & 16.000 & \multicolumn{1}{c}{13.000} & \multicolumn{1}{c}{10.000} & \multicolumn{1}{c}{0} \\
\hline $30-40$ & $3508,64 \mathrm{aA}$ & $3767,88 \mathrm{aA}$ & $3625,19 \mathrm{aA}$ & $3943,08 \mathrm{aA}$ & $3381,23 \mathrm{aA}$ \\
$20-30$ & $3815,12 \mathrm{aA}$ & $3872,40 \mathrm{aA}$ & $3330,07 \mathrm{aAB}$ & $3613,17 \mathrm{aA}$ & $2884,37 \mathrm{aB}$ \\
$10-20$ & $2691,72 \mathrm{bA}$ & $2831,83 \mathrm{bA}$ & $1993,65 \mathrm{bB}$ & $2040,70 \mathrm{bB}$ & $844,17 \mathrm{bC}$ \\
$0-10$ & $718,48 \mathrm{cA}$ & $761,61 \mathrm{cA}$ & $451,75 \mathrm{cA}$ & $634,68 \mathrm{cA}$ & $191,23 \mathrm{cA}$ \\
\hline Em cada coluna e para cada fator, médias seguidas de mesma letra minúscula e mesma letra \\
minúscula na linha não diferem pelo teste de Tukey, a 5\% de probabilidade
\end{tabular}

Para melhor visualização das variações da compactação do solo após o tráfego do pneu em função da carga aplicada, calculou-se o incremento do índice de cone para cada camada com o pneu radial e com o pneu diagonal. Pode-se observar, nas Figuras $8 \mathrm{~A}$ e B, que os maiores incrementos na compactação ocorreram nas camadas de 0 a 10 e 10 a $20 \mathrm{~cm}$, para ambos os pneus, concordando com os resultados obtidos por Ferreira (1998) e Fernandes (1996).

A. Pneu radial

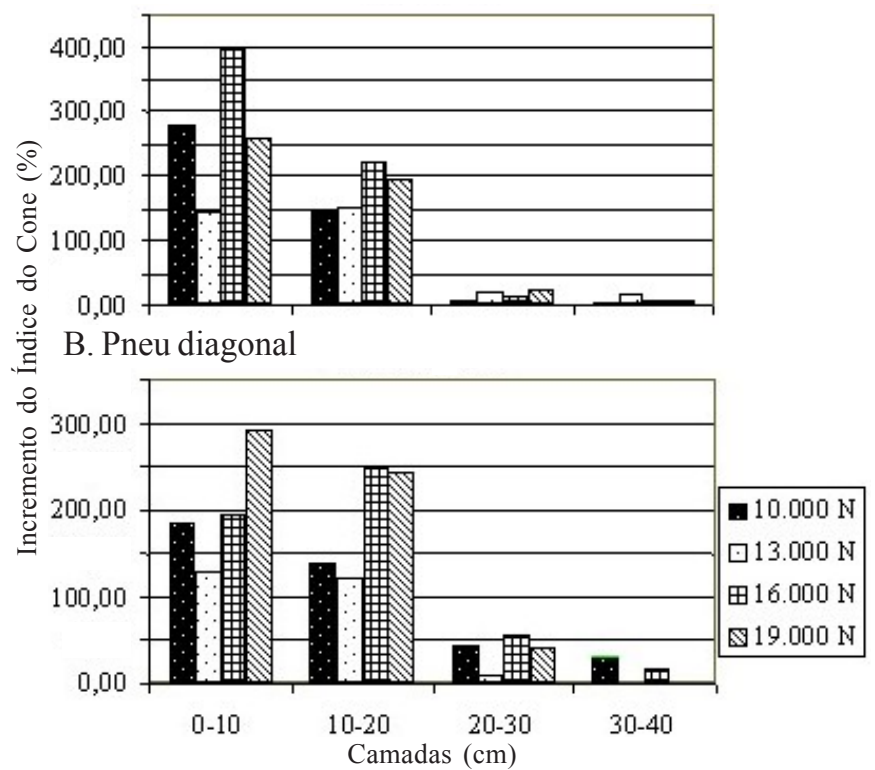

Figura 8. Comportamento das camadas do solo após o tráfego do pneu radial (A) e diagonal (B), em função da carga aplicada

Comparando-se os pneus nota-se (Figura 8) que o tráfego com o pneu radial provocou maiores incrementos na camada superficial e menores incrementos na camada inferior. Para a carga de $16.000 \mathrm{~N}$ observa-se que o incremento do índice de cone foi maior para o pneu radial. A resposta para este incremento pode estar relacionada com variações nos valores de força, torque, velocidade, resistência ao rolamento e tração líquida entre os pneus.

A partir dos resultados obtidos com o equipamento, podese estudar o comportamento do pneu para cada condição de trabalho, melhorar o desempenho do trator, aumentar a capacidade de trabalho do conjunto, evitar alterações indesejáveis na estrutura do solo, aumentar a vida útil do pneu, melhorar a qualidade do serviço executado e reduzir o custo.

\section{CONCLUSÕES}

1. Os valores de índice de cone foram maiores na camada de 10 a $20 \mathrm{~cm}$ que na camada de 0 a $10 \mathrm{~cm}$, para ambos os pneus.

2. O tipo de pneu utilizado apresentou influência significativa na compactação do solo, mostrando que, na média dos valores obtidos, o índice de cone com o pneu diagonal (2.510 $\mathrm{kPa}$ ) foi maior que com o pneu radial $(2.380 \mathrm{kPa})$.

3 . As cargas utilizadas no experimento modificaram a condição inicial do solo, apenas nas camadas de 10 a 20 e de 20 
a $30 \mathrm{~cm}$. As camadas do solo de 0 a 10 e de 10 a $20 \mathrm{~cm}$, sofreram maiores incrementos no índice de cone.

\section{LITERATURA CITADA}

Al-Adawi, S.S.; Reeder, R.C. Compaction and subsoiling effects on corn and soybean yields and soil physical properties. Transactions of ASAE, St. Joseph, v.39, p.1641-9, 1996.

Brixius, W.W. Traction prediction equations for bias ply tires. St. Joseph: ASAE, 1987. 9p. Paper 87-1622

Camargo, O.A.; Alleoni, L.R.R. Compactação do solo e desenvolvimento das plantas. Piracicaba: Degaspari, 1997. 132p.

Castro Neto, P. Desenvolvimento e avaliação de equipamentos e metodologia para determinação de parâmetros físicos do solo relacionados a dias trabalháveis com máquinas agrícolas. Botucatu, 2001. 155p. Tese Doutorado

Castro Neto, P.; Nagaoka, A.K.; Lanças, K.P. Agricultura de precisão: sistema de processamento de dados para elaboração de mapas de isocompactação. In: Simpósio em Energia na Agricultura, 1, 1999, Botucatu. Anais... Botucatu: Faculdade de Ciências Agronômicas, Universidade Estadual Paulista, 1999. v.2, p.449-53.

Cordeiro, M.A.L. Estudo do efeito de lastragem no desempenho de um tratos agrícola. Botucatu, 1994. 112p. Dissertação Mestrado

Cordeiro, M.A.L. Desempenho de um trator agrícola em função do pneu, da lastragem e da velocidade de deslocamento. Botucatu, 2000. 153p. Dissertação Mestrado

Curi, P.R. Metodologia e análise da pesquisa em ciências biológicas. Botucatu: Tiponic, 1997. 264p.

Deere, J. Optimizing your 60 and 70 series 4WD tractors for top performance. Moline: John Deere Wanterloo Works. AR 113037. 1993.22p.

EMBRAPA. Serviço Nacional de Levantamento e Conservação de Solos. Manual de métodos de análise de solos. Rio de Janeiro, 1979. Não paginado

Fernandes, H.C. Máquinas de exploração florestal: compactação das trilhas e dias potencialmente úteis para o trabalho. Botucatu, 1996. 145p. Tese Doutorado

Ferreira, M.C.D. Compactação do solo por tráfego de máquinas de colheita em um plantio florestal de Eucalyptus saligna. Piracicaba, 1998. 83p. Dissertação Mestrado
Ferreira, M.M. Física do solo. Lavras: Fundação de Apoio ao Ensino, Pesquisa e Extensão, Escola Superior de Agricultura de Lavras, 1993. 63p.

Gomes, F.P. Curso de estatística experimental. 12 ed. Piracicaba: Nobel, 1987.468p.

Goodyear Tyre \& Rubber. Farm tire handbook. Akron, 1994. 88p.

Lanças, K.P. Subsolador: constituição, regulagens, operação e desempenho. Botucatu: Faculdade de Ciências Agronômicas, Universidade Estadual Paulista, 1991. 47p.

Lanças, K.P.; Santos, C.A. Penetrômetro hidráulico-eletrônico equipado com DGPS para avaliação da compactação do solo. In: Balbuena, R.H.; Benez, S.H.; Jorajuria, D. Ingenieria rural y mecanización agrária en el ámbito latinoaméricano. La Plata: Editorial de la Universidad Nacional de La Plata, 1998. p.570576.

Marshall, R.M.; Raney, W.A. Modifying soil profiles. In: Power to produce - the yearbook of agriculture soil. Washington: United States Government. Printing Office, 1960. p.123-128.

Ortolani, A.F.; Coan, O.; Benincasa, M.; Banzato, D.A.; Gamero, C.A.; Natale, W. Manejo do solo agrícola durante dez anos com a cultura do milho (Zea mays, L.) - I Efeitos no solo. In: Congresso Brasileiro de Engenharia Agrícola, 20, 1991, Londrina. Anais... Londrina: Sociedade Brasileira de Engenharia Agrícola. 1992.p.738-62.

Seraut, A. Le pneumatique basse pression. Bulletim Tech. March. Equip. Agric. CEMAGREF. Parc de Tourvoie, Antony, n.50, p.33-37, 1990.

Souza, F.L.C.; Trein, C.R.; Campani, D.B. Determinação do perfil do solo mobilizado pela ação do subsolador. In: Congresso Brasileiro de Engenharia Agrícola, 16, 1987, Jundiaí. Anais... Jundiaí: Sociedade Brasileira de Engenharia Agrícola, 1988. p329-336.

Torres, E.; Saraiva, F.O.; Moreira, J.J.A.A.; Urchei, M.A.; Hernani, L.C.; Gaudêncio, C.A.; Primavesi, O.; Franz, C.A.B. Compactação do solo. In: Salton, J.C.; Hernani, L.C.; Fontes, C.Z. Sistema plantio direto. O produtor pergunta, a EMBRAPA responde. Dourados: Centro de Pesquisa Agropecuária Oeste-EMBRAPA, 1998. p.103-118.

Wolf, D.; Shmulevich, I.; Mussel, U. Wheel traction prediction on hard soil. Transactions of ASAE. St. Joseph, v.39, p.12751283, 1996. 